\title{
ASA404, a vascular disrupting agent, as an experimental treatment approach for brain tumors
}

\author{
OLIVER BÄHR ${ }^{1,4}$, STEFANIE GROSS ${ }^{1}$, PATRICK N. HARTER ${ }^{2,4}$, ELMAR KIRCHES $^{3}$, CHRISTIAN MAWRIN $^{3}$, \\ JOACHIM P. STEINBACH ${ }^{1,4}$ and MICHEL MITTELBRONN ${ }^{2,4-8}$ \\ ${ }^{1}$ Dr. Senckenberg Institute of Neurooncology, Goethe-University Hospital, Frankfurt; ${ }^{2}$ Institute of \\ Neurology (Edinger-Institute), Goethe-University, Frankfurt; ${ }^{3}$ Institute of Neuropathology, Otto-von-Guericke University, \\ Magdeburg; ${ }^{4}$ German Cancer Consortium (DKTK), Partner Site Frankfurt/Mainz, Frankfurt, Germany \\ and German Cancer Research Center (DKFZ), Heidelberg, Germany; ${ }^{5}$ Laboratoire National de Santé, Dudelange, \\ Luxembourg; ${ }^{6}$ Luxembourg Centre of Neuropathology (LCNP), Luxembourg City; ${ }^{7}$ Luxembourg Centre for \\ Systems Biomedicine (LCSB), University of Luxembourg, Esch-sur-Alzette; ${ }^{8}$ NORLUX Neuro-Oncology Laboratory, \\ Department of Oncology, Luxembourg Institute of Health, Strassen, Luxembourg
}

Received February 27, 2017; Accepted July 7, 2017

DOI: $10.3892 / \mathrm{ol} .2017 .6832$

\begin{abstract}
Malignant brain tumors, including gliomas, brain metastases and anaplastic meningiomas, are associated with poor prognosis, and represent an unmet medical need. ASA404 (DMXAA), a vascular disrupting agent, has demonstrated promising results in several preclinical tumor models and early phase clinical trials. However, two phase III trials in nonsmall cell lung cancer reported insufficient results. The aim of the present study was to determine the effects of ASA404 on brain tumors. The effects of ASA404 were evaluated in vitro and in vivo using subcutaneous, and orthotopical models for malignant glioma (U-87, LN-229, U-251, LN-308 and Tu-2449), brain metastasis (HT-29) and malignant meningioma (IOMM-Lee). The acute effects of ASA404 on tumor tissue were analyzed using conventional and immunohistochemical staining techniques [hematoxylin and eosin, MIB-1 antibody/ proliferation maker protein Ki-67, cleaved caspase-8, stimulator of interferon genes (STING), ionized calcium-binding adapter molecule 1]. Furthermore, the sizes of subcutaneous tumors were measured and the symptom-free survival rates of animals with intracranial tumors receiving ASA404 treatment were analyzed. ASA404 demonstrated low toxicity in vitro, but exhibited strong effects on subcutaneous tumors $24 \mathrm{~h}$ following a single dose of ASA404 (25 mg/kg). ASA404
\end{abstract}

Correspondence to: Dr Oliver Bähr, Dr. Senckenberg Institute of Neurooncology, Goethe-University Hospital Frankfurt, Schleusenweg 2-16, D-60528 Frankfurt, Germany

E-mail: oliver.baehr@med.uni-frankfurt.de

Key words: ASA404, DMXAA, vadimezan, brain tumor, glioblastoma, meningioma, brain metastases, STING, vascular disrupting agents induced necrosis, hemorrhages and inhibited the proliferation, and growth of tumors in the subcutaneous glioma models. However, ASA404 failed to demonstrate comparable effects in any of the intracranial tumor models examined and did not result in a prolongation of survival. Expression of STING, the molecular target of ASA404, and infiltration of macrophages, the cells mediating ASA404 activity, did not differ between subcutaneous and intracranial tumors. In conclusion, ASA404 demonstrates clear efficacy in subcutaneous tumor models, but has no relevant activity in orthotopic brain tumor models. The expression of STING and infiltration with macrophages were not determined to be involved in the differential activity observed among tumor models. It is possible that the low penetration of ASA-404 into the brain prevents concentrations sufficient enough reaching the tumor in order to exhibit acute effects in vivo.

\section{Introduction}

Human malignant brain tumors, glioblastomas as well as brain metastasis and anaplastic meningiomas, are highly vascularized tumors (1-4). Therefore, the tumor vasculature is a reasonable therapeutic target. To date, the VEGF neutralizing monoclonal antibody bevacizumab is the only FDA approved antiangiogenic drug for the treatment of recurrent glioblastoma $(5,6)$. However, bevacizumab failed to prolong overall survival (OS) in two phase III trials for newly diagnosed glioblastoma and in glioblastoma at first recurrence $(7,8)$. Beyond bevacizumab there has been a wealth of antiangiogenic compounds in clinical trials interfering with different pathways of angiogenesis (cilengitide, cediranib, enzastaurin, etc.) (5,9-12). ASA404 belongs to the class of vascular disrupting agents (VDA) and showed encouraging preclinical activity in a number of solid tumor models (13-20). Treatment with VDAs usually results in a rapid shut down of blood flow, resulting in extensive tumor necrosis without affecting normal blood vessels (21). In addition, ASA404 is 
able to induce an anti-tumor immunity in vivo $(18,22-25)$. Several studies showed that stimulator of interferon gene (STING) is the molecular target of ASA404, mediating the increase of inflammatory cytokines $(23,24,26-29)$. Moreover, it has been shown that only murine STING but not human STING has the ability to sense ASA404 (23,24,26,27,29-30). This might explain why ASA404 has failed in a larger clinical trial $(31,32)$. Further, tumor associated macrophages seem to be the cellular mediator of ASA404 activity $(22,33)$.

Here we report on our findings on the treatment of several subcutaneous and intracranial brain tumor models with ASA404, including colon carcinoma, malignant gliomas and meningioma.

\section{Materials and methods}

Cell lines. The malignant human glioma cell lines (U-87, LN-229, U-251, LN-308) were kindly provided by Professor N. de Tribolet (Lausanne, Switzerland). Tu-2449 is a glioma cell line derived from a spontaneous tumor in GFAP-v-src-transgenic mice and was provided by J. Weissenberger (Frankfurt, Germany) (34). HT-29 colon carcinoma cells were purchased from the American Type Culture Collection (ATCC; Manassas, VA, USA). The malignant meningioma cell line IOMM-Lee was kindly provided by David H. Gutmann (Department of Neurology, Washington University School of Medicine, St. Louis, MO, USA) (35-39). Mouse brain endothelial cells bEnd.3 immortalized with the polyoma virus middle $\mathrm{T}$ oncogene were kindly provided by Werner Risau (Max-Planck Institute for Physiological and Clinical Research, Bad Nauheim, Germany) (40-42). All cells were maintained in DMEM containing 10\% fetal calf serum, $2 \mathrm{mM}$ glutamine and penicillin $(100 \mathrm{IU} / \mathrm{ml}) /$ streptomycin $(100 \mu \mathrm{g} / \mathrm{ml})(43)$.

Reagents. ASA404 (DMXAA, vadimezan) was provided as a dry powder by Novartis (Nürnberg, Germany). ASA404 was dissolved in $50 \mathrm{mM}$ Tris buffer at a concentration of $10 \mathrm{mg} / \mathrm{ml}$ and the $\mathrm{pH}$ was adjusted to 7.8-8.2. For the used concentrations ASA404 stock solution was diluted with PBS.

Cell viability and cell death assays. For cytotoxicity assays, the cells were seeded at a density of $10^{4}$ cells/well in 96-well plates, adhered for $24 \mathrm{~h}$ and then exposed to ASA404 for $72 \mathrm{~h}$ in medium including 5\% fetal calf serum. Cell density was assessed by crystal violet staining.

Animal models. The animal studies in this study have been approved by our local authority (Regierungspräsidium, Darmstadt, Germany). Experiments have been performed in accordance with European guidelines on animal experiments and the ARRIVE guidelines.

For generation of subcutaneous tumors $3 \times 10^{6}$ tumor cells in $100 \mu 1$ PBS were injected subcutaneously in the lower back sides of 6 weeks old female athymic nude mice (Foxn1nu, Harlan, Indianapolis, IN, USA). Tumor size was evaluated twice per week using a manual caliper. Tumor volume was estimated by multiplying the three dimensions of the tumor $\left(x^{*} y^{*} \mathrm{z}\right)$ and dividing the result by 2 . The tumors were allowed to grow to a diameter of $10 \mathrm{~mm}$ before randomized treatment started. Mice were treated with a single intraperitoneal dose of ASA404 with $25 \mathrm{mg} / \mathrm{kg}$ body weight or with PBS. $24 \mathrm{~h}$ after treatment mice were sacrificed. The subcutaneous tumors were removed for further histopathologic analyses. For longitudinal tumor volumetric measurements, mice were treated twice per week.

For orthotopic tumors, $1 \times 10^{5}$ cells (U-87 and U-251) in $4 \mu 1$ PBS were injected stereotactically into the right striatum. Animals were observed daily for weight loss or neurological deterioration. At first symptoms three mice were treated with ASA404 $(25 \mathrm{mg} / \mathrm{kg})$ and three mice with PBS. After $24 \mathrm{~h}$ mice were sacrificed and brains removed for further histologic analyses. For evaluation of symptom-free survival mice were continuously treated with ASA404 $(25 \mathrm{mg} / \mathrm{kg})$ or PBS twice a week starting at day 7 after tumor implantation. When mice developed neurologic symptoms or showed a weight loss of more than $10 \%$ they were sacrificed.

As a syngeneic glioma model Tu-2449 cells were injected into the right striatum of $\mathrm{B} 6 \mathrm{C} 3 \mathrm{~F} 1$ hybrid mice (Taconic Farms, Cologne, Germany). For a brain metastasis model we stereotactically injected HT-29 cells to the right striatum and treated the animals with ASA404 at first symptoms. For a malignant meningioma model, orthotopic, subarachnoidal tumor cell inoculation with IOMM-Lee cells was done as previously described (36).

Histology/Immunohistochemistry. Subcutaneous tumors and mouse brains were formalin-fixed and paraffin-embedded. Thick sections $(8 \mu \mathrm{m})$ were cut and deparaffination procedures were performed according to standard protocols. Hematoxylin and eosin (H\&E) stainings were analyzed by experienced neuropathologists (P.N.H, M.M. and C.M.). Immunohistochemistry was carried out using a monoclonal mouse antibody against the human Ki67-antigen (clone MIB-1; Dako, Glostrup, Denmark), against cleaved caspase-3 (Asp175, 5A1E, \#9664; Cell Signaling, Leiden, The Netherlands), a polyclonal antibody against human and rodent STING (PA5-23381; Thermo Fisher, Dreieich, Germany) and a rabbit antibody against Iba1 (Wako Chemicals, Neuss, Germany) on the Ventana Discovery IHC system (Ventana, Strasbourg, France). Iba1 staining was performed using the Leica BOND III automated IHC system (Leica Biosystems, Nussloch, Germany). Only nuclear Ki67/MIB-1 staining was counted as positive. Ten high-power fields (HPF) were evaluated for the percentage of Ki67/MIB-1 positive cells.

\section{Results}

ASA404 shows low cytotoxicity in vitro. As shown in Fig. 1 ASA404 results in a reduced cell density after $72 \mathrm{~h}$ of treatment. This effect is limited to higher concentrations of ASA404 $(\geq 0.1 \mathrm{mg} / \mathrm{ml})$. No relevant differences between effects on the tumor cell lines (U-87, LN-229, HT-29) and a brain endothelial cell line (bEnd.3) were observed.

ASA404 induces large necrosis and hemorrhage in subcutaneous tumors. Acute effects of ASA404 were evaluated in subcutaneous tumor models. Tumors were excised $24 \mathrm{~h}$ after a single, intraperitoneal treatment with ASA404 $(25 \mathrm{mg} / \mathrm{kg})$ or PBS as control. All examined tumors showed large areas of 

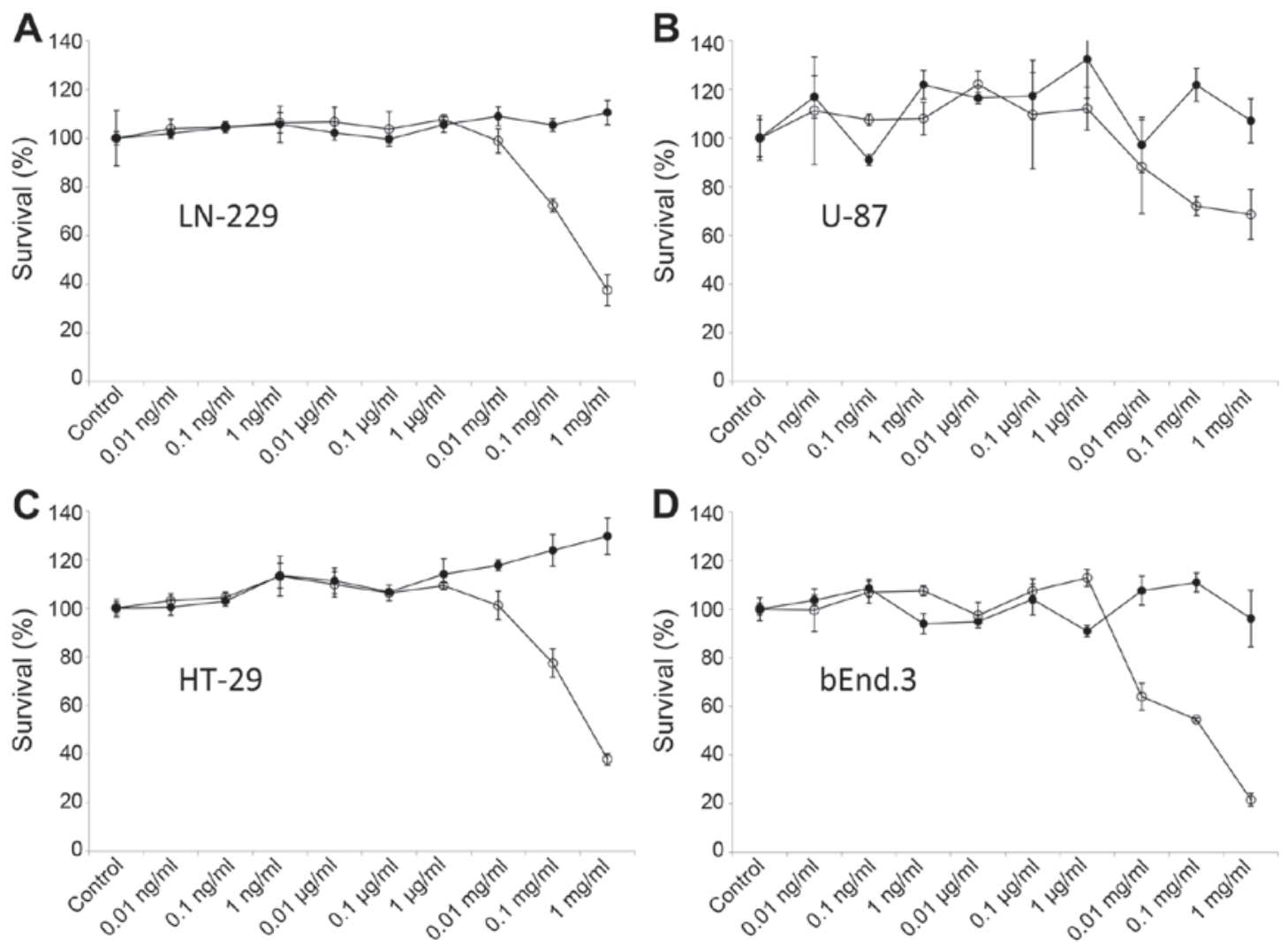

Figure 1. Cytotoxicity of ASA404 in vitro. Survival of LN-229 (A), U-87 (B), HT-29 (C) and bEnd.3 (D) cells after $72 \mathrm{~h}$ treatment with increasing concentrations of ASA404 (open circles) or solvent alone (Tris buffer, filled circles) is shown. Survival was assessed by crystal violet staining. Data are expressed as mean percentages of survival compared to control and SEM $(n=3)$.

necrotic tissue and hemorrhages shortly after treatment. Fig. 2 shows macroscopic and microscopic effects in U-87 and HT-29 tumors. Similar results have been observed in LN-229, U-251 and LN-308 tumors (data not shown).

Decreased proliferation after treatment with ASA404. We further analyzed the expression of the proliferation marker Ki67/MIB-1 in subcutaneous tumors treated with ASA404. As expected, Ki67/MIB-1 expression was not evaluable in necrotic tumor areas. Interestingly, Ki67/MIB-1 expression was markedly reduced in vital areas of the ASA404 treated tumors compared to tumors treated with PBS. As shown in Fig. 3, immunohistochemical staining for Ki67/MIB-1 in U-87 tumors treated with PBS (Fig. 3A) and treated with ASA404 (Fig. 3B). Fig. 3C and D show LN-308 tumors treated with PBS (Fig. 3C) and ASA404 (Fig. 3D). In LN-308 tumors we quantified the Ki67/MIB-1 expression by assessing the percentage of tumor cells that show nuclear expression of Ki67/MIB-1.

ASA404 induces cleavage of caspase-3 in subcutaneous tumors. As we found apoptotic figures in the necrotic areas of all subcutaneous tumors we analyzed the tumors for expression of cleaved caspase-3, an indicator for apoptosis. As shown in Fig. 4, immunohistochemistry for cleaved caspase-3 in subcutaneous U-87 tumors. The large necrotic areas on the H\&E slides (Fig. 4A and C) show strong expression of cleaved caspase-3 (Fig. 4B and D). In vital tumor areas of ASA404 treated tumors and in tumors treated with PBS cleaved caspase-3 is absent.

ASA404 dramatically reduces tumor growth in a subcutaneous glioma model. To evaluate long-term effects of ASA404 on tumor growth we continuously treated mice with subcutaneous LN-229 tumors. After all tumors reached a sufficient size (diameter 5 to $10 \mathrm{~mm}$ ) animals were randomized to receive PBS or ASA404 (25 mg/kg) twice per week. Treatment started on day 25. Animals treated with PBS showed the expected increase in tumor volume (Fig. 5A, blue line), while in animals treated with ASA404 tumor size decreased (Fig. 5A, red line). After 60 days the study was stopped and tumors excised. The mean tumor volume of PBS treated mice reached $0.96 \mathrm{~cm}^{3}$ (Fig. 5A and B, left column), while tumor volume in ASA404 treated mice reached $0.17 \mathrm{~cm}^{3}$ (Fig. 5A and B, right column).

ASA404 has no activity in orthotopic glioma models. U-87 cells were implanted stereotactically in athymic nude mice. When the first mouse showed symptoms all animals were randomized to receive either PBS (Fig. 6A and C) or ASA404 (25 mg/kg, Fig. 6B and D) intraperitoneally. $24 \mathrm{~h}$ after treatment mice were sacrificed and brains excised. We did not find relevant differences on H\&E slides between PBS and ASA404 treated tumors (Fig. 6A-D). Especially, we did not find necrotic areas or an increase in intratumoral hemorrhage. Identical results were found for U-251 and Tu-2449 tumors. 


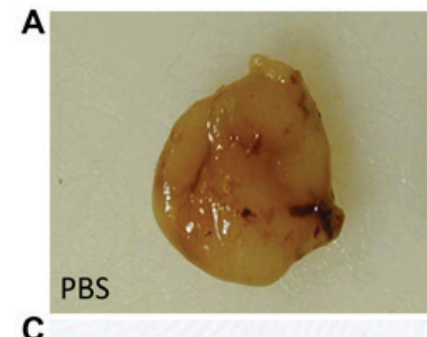

C
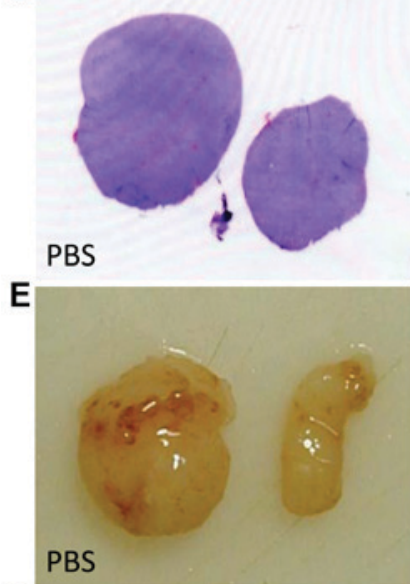

G

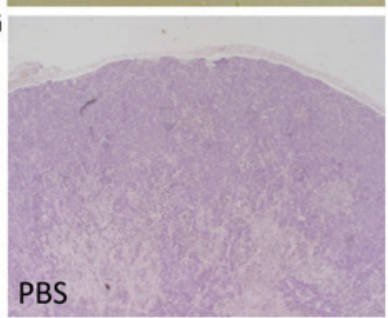

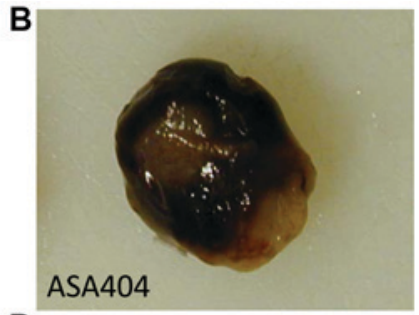

D
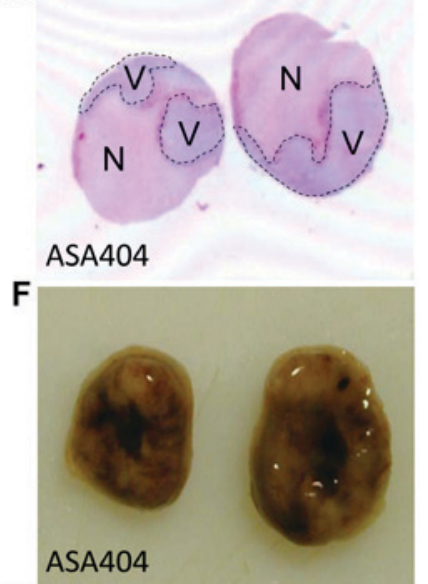

H

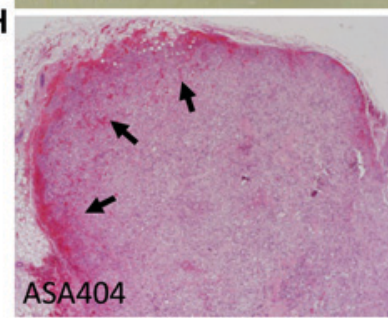

Figure 2. Acute effects of ASA404 treatment in subcutaneous tumors. (A-D) show acute effects ( $24 \mathrm{~h}$ ) of ASA404 treatment in subcutaneous U-87 tumors. A and $\mathrm{C}$ show control tumors treated with PBS. B and D show subcutaneous U-87 tumors $24 \mathrm{~h}$ after treatment with ASA404 $(25 \mathrm{mg} / \mathrm{kg}$ body weight). (A and B) Macroscopic images of the tumors after excision. (C and D) Overview on the H\&E staining with large areas of necrotic tissue (N, necrosis; V, vital tumor). (E and F) show images of HT-29 tumors ( $\mathrm{G}$ and $\mathrm{H}$ ) show histological images of $\mathrm{H} \& \mathrm{E}$ staining with marked hemorrhages (arrows).

Further, we evaluated the influence of ASA404 on symptom-free survival. Athymic nude mice with U-87 tumors were continuously treated with PBS or ASA404 twice a week starting at day 7 after tumor implantation. Median survival for PBS was 36 days (Fig. 6E, blue line) and for ASA404 40 days (Fig. 6E, red line).

ASA404 has no activity in models for CNS metastasis and malignant meningioma. To further evaluate the brain activity of ASA404 we applied an animal model for CNS metastasis using the colon carcinoma cell line HT-29 injected to the right striatum. As a meningioma model the malignant meningioma cell line IOMM-Lee was inoculated into the subarachnoid space. Mice were sacrificed $24 \mathrm{~h}$ after treatment with PBS or ASA404 (25 mg/kg) and brains/tumors excised for histological analyses (Fig. 7). As shown in Fig. 7A and C (PBS treated mice) HT-29 cells formed large and necrotic tumors. After treatment with ASA404 (Fig. 7B and D) viability was not reduced and we did not see additional necrotic areas, suggesting that ASA404 had no activity in this model. No obvious activity
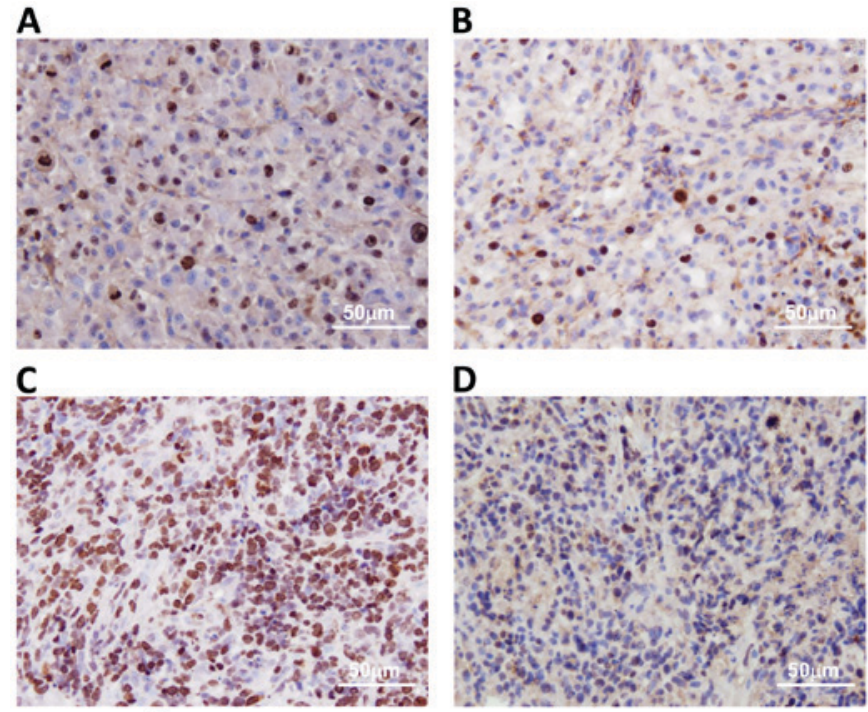

D

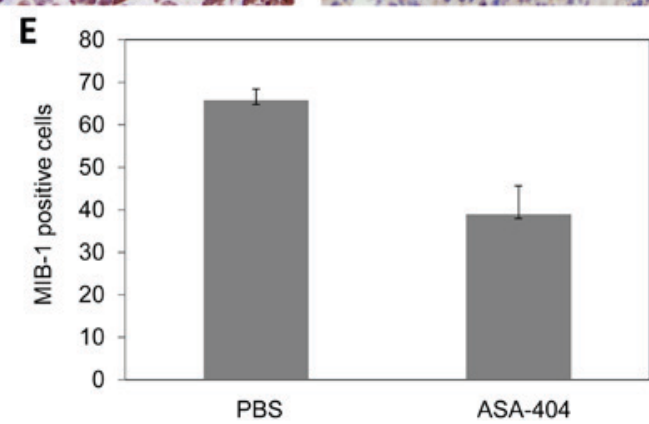

Figure 3. Expression of the proliferation marker Ki67/MIB-1 in subcutaneous tumors treated with ASA404. Subcutaneous U-87 (A and B) and LN-308 (C and D) tumors are shown. A and C shows expression of Ki67/MIB-1 in tumors treated with PBS and B and D shows tumors treated with ASA404. The percentage of Ki67/MIB-1 nuclear positive tumor cells was evaluated in 10 high power fields in subcutaneous LN-308 tumors treated with PBS (E, left bar) or ASA404 (E, right bar).

of ASA404 has been detected in the malignant meningioma model (Fig. 7E and G shows PBS treatment, Fig. 7F and $\mathrm{H}$ shows ASA404 treatment).

STING expression and ibal positive cells in subcutaneous and intracranial U-87 tumors. As STING is the molecular target of ASA404 in a murine setting we examined tumor tissue for STING expression using a monoclonal antibody recognizing both human and murine STING. As shown in Fig. 8 STING expression in a subcutaneous (Fig. 8A) and an intracranial (Fig. 8B) U-87 tumor. We did not find a clear difference in STING expression explaining the differential activity of ASA404. Notably, STING expression was more focused in the intracranial tumor, while most cells in the subcutaneous tumor showed a more widespread, cytosolic STING expression.

As macrophages in the tumor microenvironment are recognized as the mediator cells of STING activity we used ibal immunohistochemistry to stain for macrophages and microglia. Again no relevant differences were observed between subcutaneous (Fig. 8C) and intracranial (Fig. 8D) tumors. We found a high number of ibal positive cells in both, subcutaneous and intracranial tumors. 
A

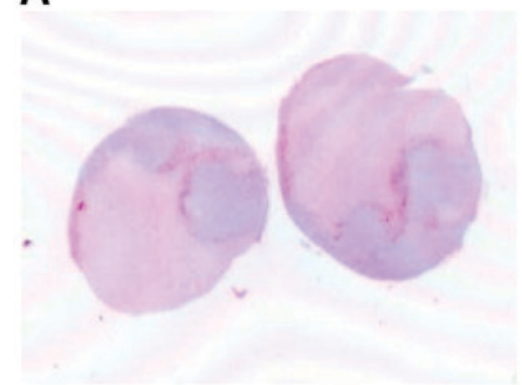

C

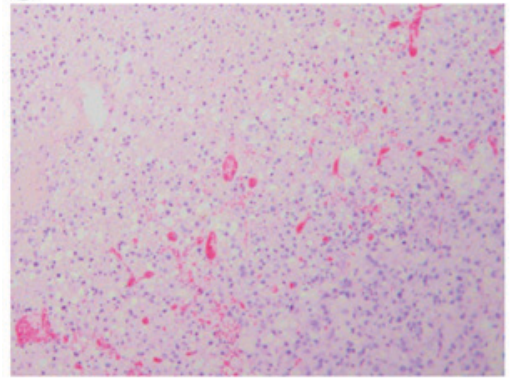

B

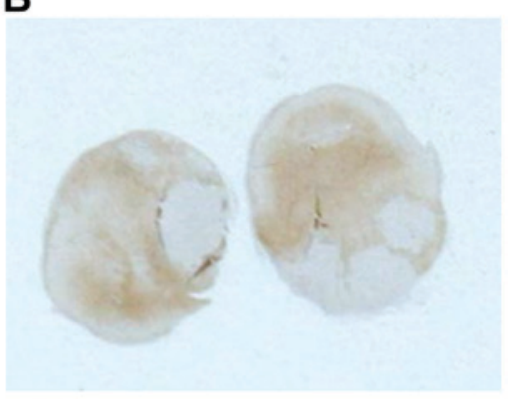

D

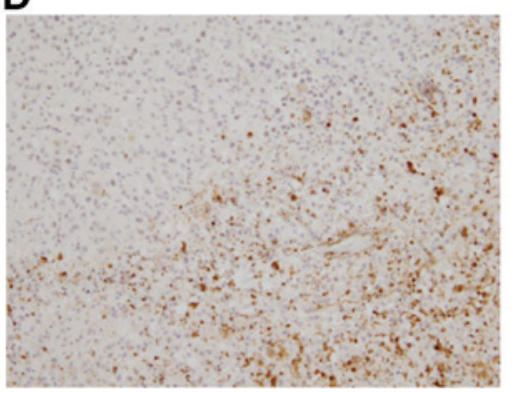

Figure 4. Expression of cleaved caspase-8 in subcutaneous tumors treated with ASA404. H\&E slides (A and C) and immunohistochemistry for cleaved caspase-8 (B and D) in subcutaneous U-87 tumors is shown. The large necrotic areas on the H\&E slides (A and C), show strong expression of cleaved caspase-8 (B and D). In vital tumor areas cleaved caspase-8 is absent.

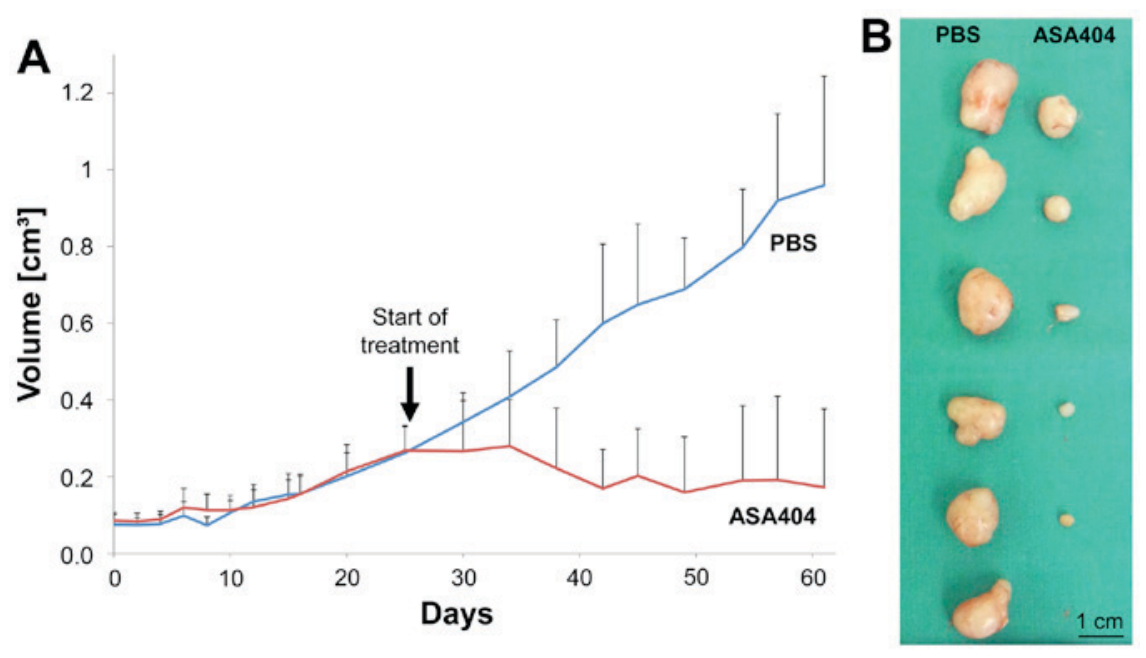

Figure 5. ASA404 reduces tumor growth in a subcutaneous glioma model. Volume of subcutaneous LN-229 tumors is shown in (A). Treatment with PBS (blue line) or ASA404 (red line) was started on day 25 ( $n=6$, mean volume, SEM). After 60 days the study was stopped and the tumors excised (B).

\section{Discussion}

More efficacious therapies are desperately needed for patients with malignant brain tumors, in particular for glioblastoma. One new approach is using VDA. ASA404, or DMXAA/vadimezan, is a vascular disrupting agent that has shown preclinical activity in several different tumor models (17-20). Earlier phase clinical trials showed promising results, but a phase III trial in non-small cell lung cancer was negative $(31,32)$. Preclinical studies on the efficacy of ASA404 in brain tumors are rare, clinical trials do not exist $(20,44)$.

In the current study we can show that ASA404 has moderate to low in vitro activity in human glioma cell lines
(U-87, LN-229), a colon carcinoma cell line (HT-29) and a brain endothelial cell line (bEnd.3). In contrast, ASA404 has dramatic effects in several subcutaneous glioma models (U-87, LN-229, U-251 and LN-308) and a colon carcinoma model (HT-29). Treatment with ASA404 resulted in extensive necrosis and hemorrhages within a few hours. When applied continuously ASA404 lead to a marked reduction of tumor growth. Unfortunately, these impressive results did not translate to the orthotopic, intracerebral glioma models (U-87, U-251). To evaluate whether this could be a cell type specific issue, we evaluated ASA404 in a murine, syngeneic glioma model (Tu-2449), a model for CNS metastasis (HT-29) and a model for malignant meningioma (IOMM-Lee). Again, 
A

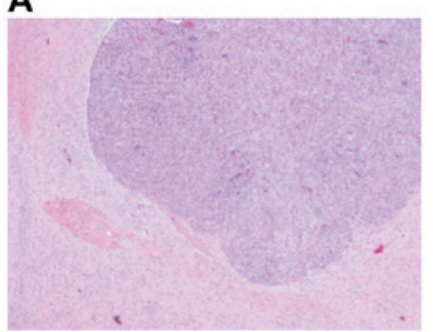

C

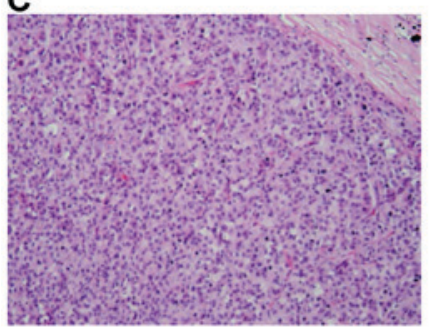

B

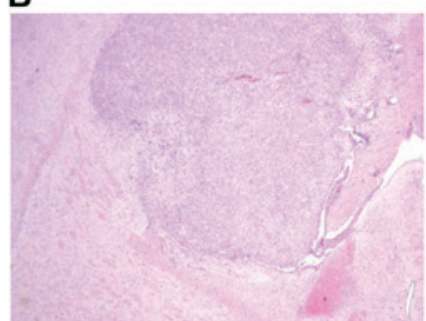

D
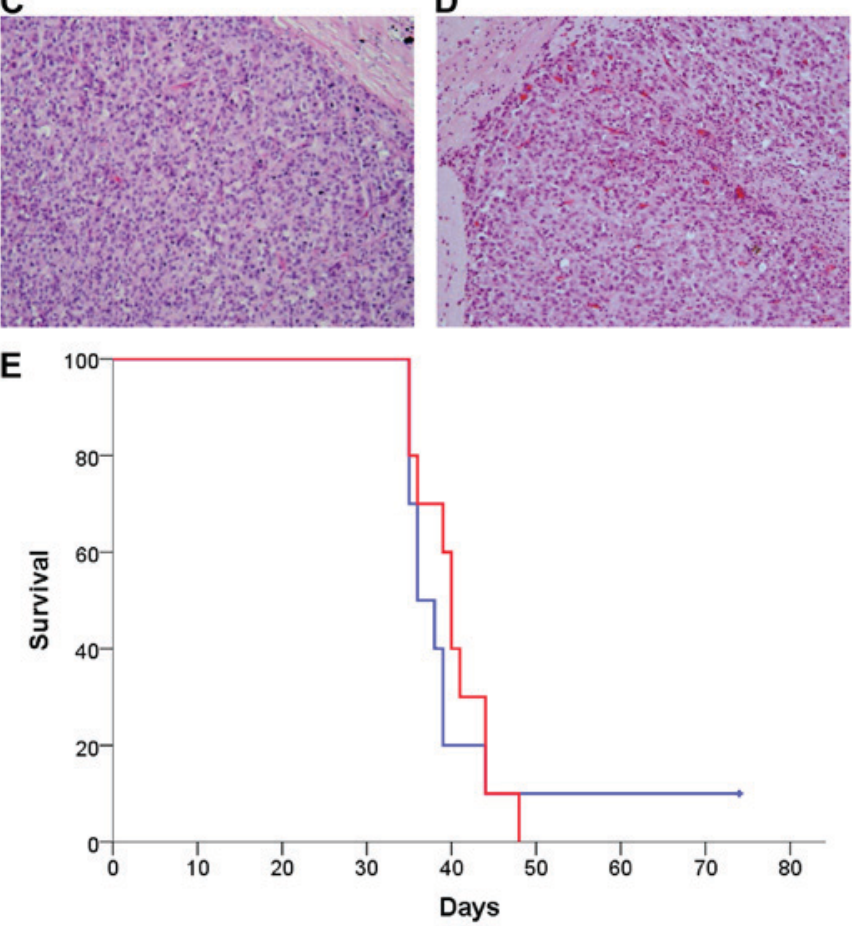

Figure 6. ASA404 in an orthotopic glioma model. H\&E staining of an orthotopic U-87 tumor $24 \mathrm{~h}$ after treatment with PBS (A and C) or ASA404 (B and D) are shown. Influence of ASA404 on symptom-free survival is shown (E). Mice with orthotopic U-87 tumors were randomized to receive PBS (blue line) or ASA404 (red line) on day 20 and day 30 after tumor implantation. Tick marks indicate censored mice.

ASA404 was not able to induce the strong effects seen in the subcutaneous models. In summary, we report on diverging effects in subcutaneous and intracranial tumor models.

In general, this once again illustrates that subcutaneous animal models are usually futile for brain tumor research and should be limited to specific mechanistic issues.

Several explanations are conceivable for our diverging effects. First, it has been shown that ASA404 does not cross the blood-brain-barrier to a relevant degree in a syngeneic glioma mouse model using the GL261 glioma cell line and C57BL/6 mice (44). At a dose of $25 \mathrm{mg} / \mathrm{kg}$ ASA404 reached a $\mathrm{C}_{\max }$ of $474 \mu \mathrm{mol} / 1$ in the plasma of tumor-bearing mice. In contrast, $\mathrm{C}_{\max }$ in the brain of tumor-bearing mice only reached $6.9 \mu \mathrm{mol} / 1$. For this pharmacokinetic analysis whole brain lysates but not brain tumor lysates were used (44). Further, the GL261 tumors only show minor contrast enhancement on MRI scans suggesting only a mild disruption of the blood-brain-barrier (45). In other tumor models and the clinical situation the blood-brain-barrier in malignant brain tumors is rather markedly dysfunctional and ASA404 might reach higher concentrations in tumor tissue. However, this
A

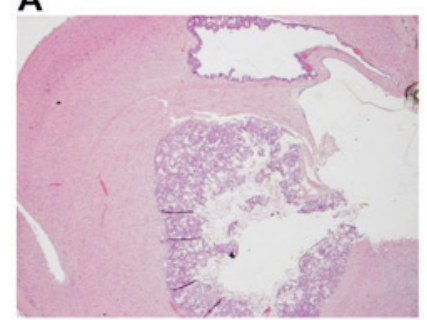

C

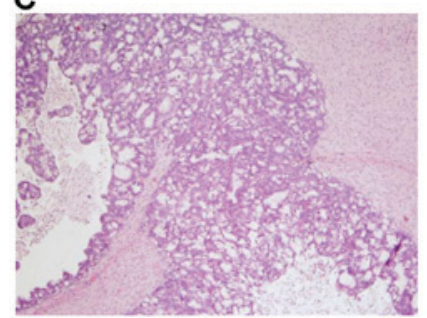

E

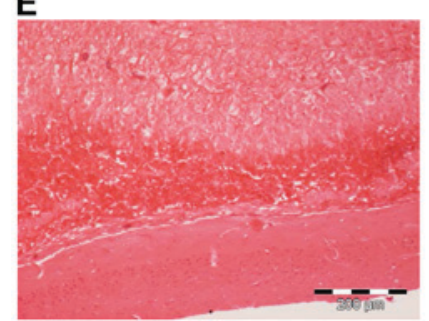

G

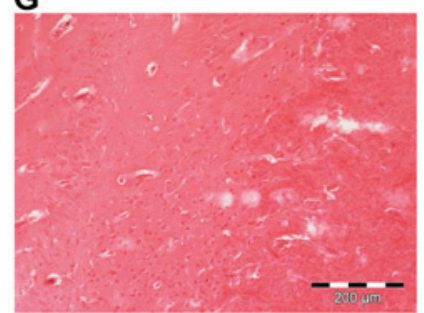

B

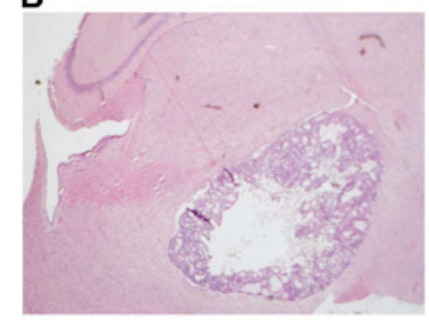

D

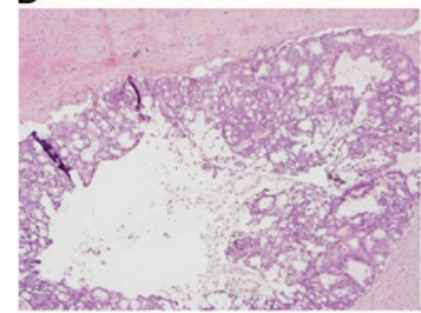

F

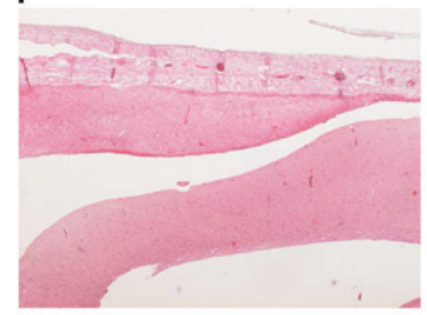

H

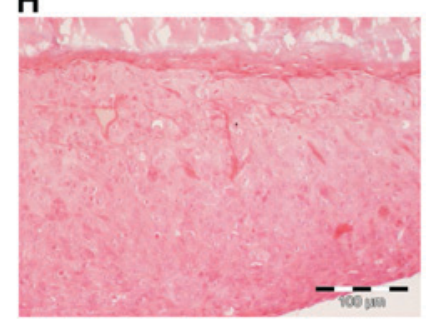

Figure 7. ASA404 in models for CNS metastasis and malignant meningioma. H\&E stainings of intracerebral HT-29 tumors (colon carcinoma) treated with PBS (A and C) or ASA404 (B and D) are shown. Intracranial IOMM-Lee tumors (malignant meningioma) treated with PBS (E and G) or ASA404 ( $\mathrm{F}$ and $\mathrm{H}$ ) are shown in the lower panel.

issue of limited brain penetration still might be the explanation why ASA404 did not show intracranial activity in our models. In contrast to our results, ASA404 increased survival in a previous study using orthotopic the GL261 and the U-87 mouse model (20). In both models only minimal contrast enhancement was detected before treatment. Notably, in both models marked extravasation of the contrast agent was detected $24 \mathrm{~h}$ after treatment with ASA404. A more recent study did not find increased survival with ASA404 in an orthotopic GL261 model corroborating our results (44).

Second, there might be a difference in molecular and/or cellular effectors. In 2005 it has been shown that tumor associated macrophages are the main cellular target of ASA404 $(22,33)$. Moreover, the STING has been identified as the molecular target of ASA404 recently $(23,24,26,27,29,30)$. Interestingly, only murine STING binds to ASA404 and leads to TANK-binding kinase 1 and IFN regulatory factor 3 signaling $(25,29)$. Human STING failed to bind to or signal in response to ASA404, which might be the explanation 

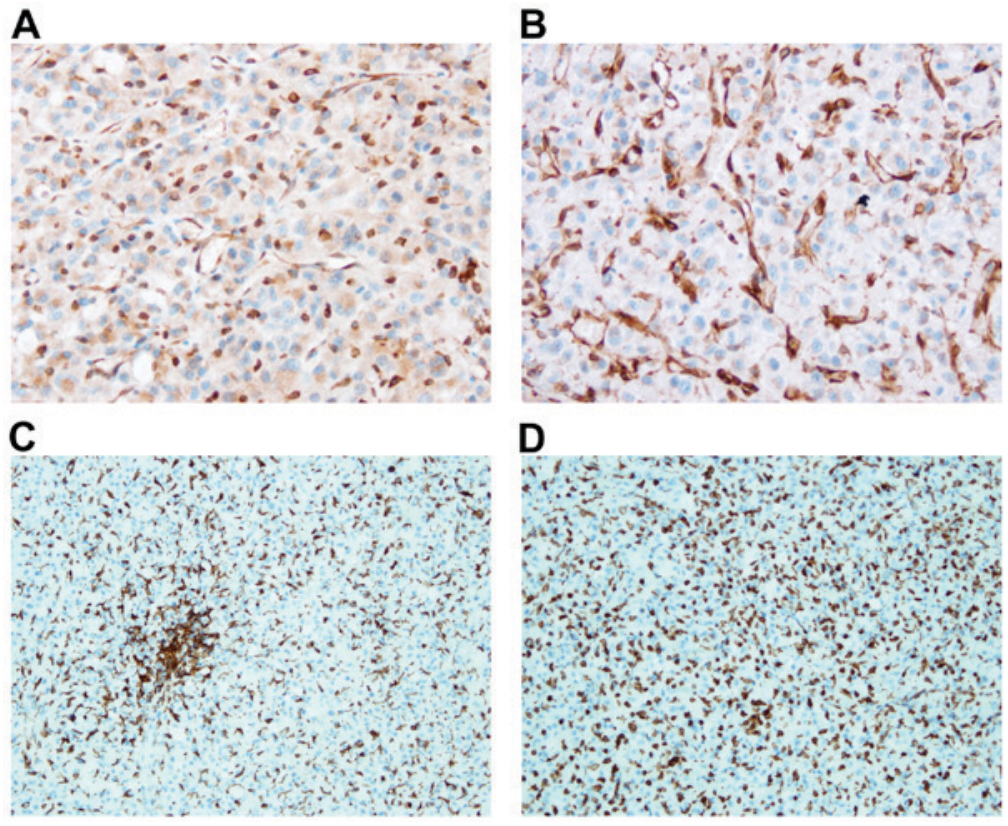

Figure 8. STING expression and iba1 positive cells in subcutaneous and intracranial U-87 tumors. Immunohistochemistry for STING is shown for subcutaneous (A) and intracranial (B) U-87 tumors. Cells positive for iba1 are stained in subcutaneous (C) and intracranial (D) U-87 tumors.

why ASA404 failed in clinical trials for human cancer (29). These findings would make it essential that a relevant number of murine tumor associated macrophages are present in a tumor to make it susceptible to ASA404. Therefore, we screened tumor tissue for STING expression and tumor associated macrophages. As shown in Fig. 8 we did not detect fundamental differences in STING expression between subcutaneous (Fig. 8A) and intracranial tumors (Fig. 8B). Whether the more diffuse and more cytosolic expression of STING in subcutaneous tumors has relevance, is beyond the scope of this study. STING expression in intracranial tumors (Fig. 8B) seemed to be more focused and restricted to fewer cells. Moreover, we were not able to specifically detect murine STING as available antibodies either recognize human and murine STING or just human STING. In the light of the brain penetration issues further evaluations on this topic might be of limited interest. Regarding tumor associated macrophages we used an antibody against ibal to detect macrophages in the examined tumor tissue. Obviously, ibal can be found on macrophages and microglia, impeding differentiation between macrophages and microglia particularly in the intracranial tumors. Again we did not find considerable differences in the number of ibal positive cells in the examined tumor tissue from subcutaneous (Fig. 8C) and intracranial (Fig. 8D) tumor tissue.

In summary, we can show that ASA404 (DMXAA, vadimezan) leads to strong responses in the subcutaneous setting when using several human glioma cell lines and one colon carcinoma cell line in athymic mice. This is of particular importance, regarding STING, the molecular target of ASA404. It has been shown that ASA404 only binds to the murine form of STING and not to human STING. Therefore, effects of ASA404 cannot directly be conveyed via human tumor cells. On the one hand, this might be an explanation for failure of ASA404 in later stage clinical trials. On the other hand, this emphasizes the importance of tumor infiltrating host cells in our setting with human cancer cells growing in a rodent host. This renders host macrophages as a promising and untransformed therapeutic target. Current knowledge on vascular disrupting properties of ASA404 suggests that tumor infiltrating macrophages sense ASA404 via STING and then initiate the release of cytokines leading to disrupted blood vessels and hemorrhagic necrosis. Unfortunately, activity of ASA404 was limited to the subcutaneous setting and we did not detect changes in several orthotopic brain tumor models. This might be due to a different cellular composition of the tumor microenvironment, but most likely is the result of the missing ability of ASA404 to cross the blood-brain-barrier (46).

Besides vascular disruption, STING agonists also lead to an activation of the innate immunity (24). STING activation results in type I interferon production and thereby activating dendritic cells in the tumor and augmenting the priming of tumor-specific T cells. Further, STING activation enhances recruitment of effector $\mathrm{T}$ cells into the tumor microenvironment $(22-24,27,29,30,33,47)$. Several synthetic agonists of human STING have been developed as immunotherapeutics and at least one is currently tested in a phase 1 trial (NCT02675439) $(23,24,26)$. Whether these new STING agonists cross the blood-brain-barrier has not been demonstrated so far. Nonetheless, agonists for human STING are highly promising new drugs as VDA and more importantly as immunotherapeutics.

\section{Acknowledgements}

This work has been partially supported by an unrestricted Grant from Novartis. The Dr. Senckenberg Institute of Neurooncology is supported by the Dr. Senckenberg Foundation. M.M. would like to thank the Luxembourg National Research Fond (FNR) for the support (FNR PEARL P16/BM/11192868 grant). O.B. 
has served as a consultant for Roche, BMS and NOXXON and has received travel grants from Roche, BMS and medac. J.P.S. has received a grant from Merck as well as honoraria for lectures, travel or advisory board participation from Roche, Boehringer, Bristol-Myers Squibb, Medac and Mundipharma.

\section{References}

1. Carmeliet P and Jain RK: Molecular mechanisms and clinical applications of angiogenesis. Nature 473: 298-307, 2011.

2. Louis DN, Perry A, Reifenberger G, von Deimling A, Figarella-Branger D, Cavenee WK, Ohgaki H, Wiestler OD, Kleihues P and Ellison DW: The 2016 world health organization classification of tumors of the central nervous system: A summary. Acta Neuropathol 131: 803-820, 2016.

3. Preusser M, Hassler M, Birner P, Rudas M, Acker T, Plate KH, Widhalm G, Knosp E, Breitschopf H, Berger J and Marosi C: Microvascularization and expression of VEGF and its receptors in recurring meningiomas: Pathobiological data in favor of antiangiogenic therapy approaches. Clin Neuropathol 31: 352-360, 2012.

4. Takano S: Glioblastoma angiogenesis: VEGF resistance solutions and new strategies based on molecular mechanisms of tumor vessel formation. Brain Tumor Pathol 29: 73-86, 2012.

5. Batchelor TT, Reardon DA, de Groot JF, Wick W and Weller M: Antiangiogenic therapy for glioblastoma: Current status and future prospects. Clin Cancer Res 20: 5612-5619, 2014.

6. Friedman HS, Prados MD, Wen PY, Mikkelsen T, Schiff D, Abrey LE, Yung WK, Paleologos N, Nicholas MK, Jensen R, et al: Bevacizumab alone and in combination with irinotecan in recurrent glioblastoma. J Clin Oncol 27: 4733-4740, 2009.

7. Chinot OL, Wick W, Mason W, Henriksson R, Saran F, Nishikawa R, Carpentier AF, Hoang-Xuan K, Kavan P, Cernea D, et al: Bevacizumab plus radiotherapy-temozolomide for newly diagnosed glioblastoma. N Engl J Med 370: 709-722, 2014.

8. Gilbert MR, Dignam JJ, Armstrong TS, Wefel JS, Blumenthal DT, Vogelbaum MA, Colman H, Chakravarti A, Pugh S, Won M, et al: A randomized trial of bevacizumab for newly diagnosed glioblastoma. N Engl J Med 370: 699-708, 2014.

9. Batchelor TT, Mulholland P, Neyns B, Nabors LB, Campone M, Wick A, Mason W, Mikkelsen T, Phuphanich S, Ashby LS, et al: Phase III randomized trial comparing the efficacy of cediranib as monotherapy and in combination with lomustine, versus lomustine alone in patients with recurrent glioblastoma. J Clin Oncol 31: 3212-3218, 2013.

10. Wick W, Puduvalli VK, Chamberlain MC, van den Bent MJ, Carpentier AF, Cher LM, Mason W, Weller M, Hong S, Musib L, et al: Phase III study of enzastaurin compared with lomustine in the treatment of recurrent intracranial glioblastoma. J Clin Oncol 28: 1168-1174, 2010.

11. Wick W, Steinbach JP, Platten M, Hartmann C, Wenz F, von Deimling A, Shei P, Moreau-Donnet V, Stoffregen C and Combs SE: Enzastaurin before and concomitant with radiation therapy, followed by enzastaurin maintenance therapy, in patients with newly diagnosed glioblastoma without MGMT promoter hypermethylation. Neuro Oncol 15: 1405-1412, 2013.

12. Stupp R, Hegi ME, Gorlia T, Erridge SC, Perry J, Hong YK, Aldape KD, Lhermitte B, Pietsch T, Grujicic D, et al: Cilengitide combined with standard treatment for patients with newly diagnosed glioblastoma with methylated MGMT promoter (CENTRIC EORTC 26071-22072 study): A multicentre, randomised, open-label, phase 3 trial. Lancet Oncol 15: 1100-1108, 2014.

13. Baguley BC: Antivascular therapy of cancer: DMXAA. Lancet Oncol 4: 141-148, 2003.

14. Hinnen $P$ and Eskens FA: Vascular disrupting agents in clinical development. Br J Cancer 96: 1159-1165, 2007.

15. Thorpe PE: Vascular targeting agents as cancer therapeutics. Clin Cancer Res 10: 415-427, 2004.

16. Tozer GM, Kanthou C and Baguley BC: Disrupting tumour blood vessels. Nat Rev Cancer 5: 423-435, 2005.

17. Folaron M, Kalmuk J, Lockwood J, Frangou C, Vokes J, Turowski SG, Merzianu M, Rigual NR, Sullivan-Nasca M, Kuriakose MA, et al: Vascular priming enhances chemotherapeutic efficacy against head and neck cancer. Oral Oncol 49: 893-902, 2013
18. Kanwar JR,KanwarRK,Pandey S, Ching LM and Krissansen GW: Vascular attack by 5,6-dimethylxanthenone-4-acetic acid combined with B7.1 (CD80)-mediated immunotherapy overcomes immune resistance and leads to the eradication of large tumors and multiple tumor foci. Cancer Res 61: 1948-1956, 2001.

19. Matthews KE, Hermans IF, Roberts JM, Ching LM and Ronchese F: 5,6-Dimethylxanthenone-4-acetic acid treatment of a non-immunogenic tumour does not synergize with active or passive $\mathrm{CD} 8^{+} \mathrm{T}$-cell immunotherapy. Immunol Cell Biol 84: 383-389, 2006.

20. Seshadri M and Ciesielski MJ: MRI-based characterization of vascular disruption by 5,6-dimethylxanthenone-acetic acid in gliomas. J Cereb Blood Flow Metab 29: 1373-1382, 2009.

21. Ching LM, Zwain S and Baguley BC: Relationship between tumour endothelial cell apoptosis and tumour blood flow shutdown following treatment with the antivascular agent DMXAA in mice. Br J Cancer 90: 906-910, 2004.

22. Jassar AS, Suzuki E, Kapoor V, Sun J, Silverberg MB, Cheung L, Burdick MD, Strieter RM, Ching LM, Kaiser LR, et al: Activation of tumor-associated macrophages by the vascular disrupting agent 5,6-dimethylxanthenone-4-acetic acid induces an effective $\mathrm{CD}^{+} \mathrm{T}$-cell-mediated antitumor immune response in murine models of lung cancer and mesothelioma. Cancer Res 65: 11752-11761, 2005.

23. Corrales L, Glickman LH, McWhirter SM, Kanne DB, Sivick KE, Katibah GE, Woo SR, Lemmens E, Banda T, Leong JJ, et al: Direct activation of STING in the tumor microenvironment leads to potent and systemic tumor regression and immunity. Cell Rep 11: 1018-1030, 2015.

24. Corrales L, McWhirter SM, Dubensky TW Jr and Gajewski TF: The host STING pathway at the interface of cancer and immunity. J Clin Invest 126: 2404-2411, 2016.

25. Roberts ZJ, Goutagny N, Perera PY, Kato H, Kumar H, Kawai T, Akira S, Savan R, van Echo D, Fitzgerald KA, et al: The chemotherapeutic agent DMXAA potently and specifically activates the TBK1-IRF-3 signaling axis. J Exp Med 204: 1559-1569, 2007.

26. Curran E, Chen X, Corrales L, Kline DE, Dubensky TW Jr, Duttagupta P, Kortylewski M and Kline J: STING pathway activation stimulates potent immunity against acute myeloid leukemia. Cell Rep 15: 2357-2366, 2016.

27. Gao P, Ascano M, Zillinger T, Wang W, Dai P, Serganov AA, Gaffney BL, Shuman S, Jones RA, Deng L, et al: Structure-function analysis of STING activation by c[G $\left(2^{\prime}, 5^{\prime}\right) \mathrm{pA}$ $\left(3^{\prime}, 5^{\prime}\right)$ p] and targeting by antiviral DMXAA. Cell 154: 748-762, 2013.

28. Ishikawa $\mathrm{H}$ and Barber GN: STING is an endoplasmic reticulum adaptor that facilitates innate immune signalling. Nature 455: 674-678, 2008

29. Conlon J, Burdette DL, Sharma S, Bhat N, Thompson M, Jiang Z, Rathinam VA, Monks B, Jin T, Xiao TS, et al: Mouse, but not human STING, binds and signals in response to the vascular disrupting agent 5,6-dimethylxanthenone-4-acetic acid. J Immunol 190: 5216-5225, 2013

30. Prantner D, Perkins DJ, Lai W, Williams MS, Sharma S, Fitzgerald KA and Vogel SN: 5,6-Dimethylxanthenone-4-acetic acid (DMXAA) activates stimulator of interferon gene (STING)-dependent innate immune pathways and is regulated by mitochondrial membrane potential. J Biol Chem 287: 39776-39788, 2012.

31. McKeage MJ, Von Pawel J, Reck M, Jameson MB, Rosenthal MA, Sullivan R, Gibbs D, Mainwaring PN, Serke M, Lafitte JJ, et al: Randomised phase II study of ASA404 combined with carboplatin and paclitaxel in previously untreated advanced non-small cell lung cancer. Br J Cancer 99: 2006-2012, 2008.

32. Lara PN Jr, Douillard JY, Nakagawa K, von Pawel J, McKeage MJ, Albert I, Losonczy G, Reck M, Heo DS, Fan X, et al: Randomized phase III placebo-controlled trial of carboplatin and paclitaxel with or without the vascular disrupting agent vadimezan (ASA404) in advanced non-small-cell lung cancer. J Clin Oncol 29: 2965-2971, 2011.

33. Wallace A, LaRosa DF, Kapoor V, Sun J, Cheng G, Jassar A, Blouin A, Ching LM and Albelda SM: The vascular disrupting agent, DMXAA, directly activates dendritic cells through a MyD88-independent mechanism and generates antitumor cytotoxic T lymphocytes. Cancer Res 67: 7011-7019, 2007.

34. Smilowitz HM, Weissenberger J, Weis J, Brown JD, O'Neill RJ and Laissue JA: Orthotopic transplantation of v-src-expressing glioma cell lines into immunocompetent mice: Establishment of a new transplantable in vivo model for malignant glioma. J Neurosurg 106: 652-659, 2007. 
35. Tuchen M, Wilisch-Neumann A, Daniel EA, Baldauf L, Pachow D, Scholz J, Angenstein F, Stork O, Kirches E and Mawrin C: Receptor tyrosine kinase inhibition by regorafenib/sorafenib inhibits growth and invasion of meningioma cells. Eur J Cancer 73: 9-21, 2017.

36. Pachow D, Andrae N, Kliese N, Angenstein F, Stork O, Wilisch-Neumann A, Kirches E and Mawrin C: mTORC1 inhibitors suppress meningioma growth in mouse models. Clin Cancer Res 19: 1180-1189, 2013.

37. McCutcheon IE, Friend KE, Gerdes TM, Zhang BM, Wildrick DM and Fuller GN: Intracranial injection of human meningioma cells in athymic mice: An orthotopic model for meningioma growth. J Neurosurg 92: 306-314, 2000.

38. Lee WH: Characterization of a newly established malignant meningioma cell line of the human brain: IOMM-Lee. Neurosurgery 27: 389-396, 1990.

39. Kliese N, Gobrecht P, Pachow D, Andrae N, Wilisch-Neumann A, Kirches E, Riek-Burchardt M, Angenstein F, Reifenberger G, Riemenschneider M, et al: miRNA-145 is downregulated in atypical and anaplastic meningiomas and negatively regulates motility and proliferation of meningioma cells. Oncogene 32: 4712-4720, 2013

40. RayChaudhury A, Frazier WA and D'Amore PA: Comparison of normal and tumorigenic endothelial cells: Differences in thrombospondin production and responses to transforming growth factor-beta. J Cell Sci 107: 39-46, 1994

41. Muhlner U, Mohle-Steinlein U, Wizigmann-Voos S, Christofori G, Risau W and Wagner EF: Formation of transformed endothelial cells in the absence of VEGFR-2/Flk-1 by Polyoma middle T oncogene. Oncogene 18: 4200-4210, 1999

42. Montesano R, Pepper MS, Möhle-Steinlein U, Risau W, Wagner EF and Orci L: Increased proteolytic activity is responsible for the aberrant morphogenetic behavior of endothelial cells expressing the middle T oncogene. Cell 62: 435-445, 1990.
43. Weller M, Rieger J, Grimmel C, Van Meir EG, Tribolet N, De Krajewski S, Reed JC, von Deimling A and Dichgans J: Predicting chemoresistance in human malignant glioma cells: The role of molecular genetic analyses. Int J Cancer 79: 640-644, 1998.

44. Yung R, Seyfoddin V, Guise C, Tijono S, McGregor A, Connor B and Ching LM: Efficacy against subcutaneous or intracranial murine GL261 gliomas in relation to the concentration of the vascular-disrupting agent, 5,6-dimethylxanthenone-4-acetic acid (DMXAA), in the brain and plasma. Cancer Chemother Pharmacol 73: 639-649, 2014.

45. Renner DN, Jin F, Litterman AJ, Balgeman AJ, Hanson LM, Gamez JD, Chae M, Carlson BL, Sarkaria JN, Parney IF, et al: Effective treatment of established GL261 murine gliomas through picornavirus vaccination-enhanced tumor antigen-specific $\mathrm{CD} 8^{+}$ T cell responses. PLoS One 10: e0125565, 2015.

46. Zhao L, Ching LM, Kestell P and Baguley BC: The antitumour activity of 5,6-dimethylxanthenone-4-acetic acid (DMXAA) in TNF receptor-1 knockout mice. Br J Cancer 87: 465-470, 2002.

47. Conlon J, Burdette DL, Sharma S, Bhat N, Thompson M, Jiang Z, Rathinam VA, Monks B, Jin T, Xiao TS, et al: Mouse, but not human STING, binds and signals in response to the vascular disrupting agent 5,6-dimethylxanthenone-4-acetic acid. J Immunol 190: 5216-5225, 2013. 